\title{
INTELIGENCIA EMOCIONAL: EVALUACIÓN Y ESTRATEGIAS EN TIEMPOS DE PANDEMIA
}

\section{EMOTIONAL INTELLIGENCE: EVALUATION AND STRATEGIES IN TIMES OF PANDEMIC}

\author{
Mateo Fabián Ortiz Mancero ${ }^{1}$ \\ Aracelly Fernanda Núñez Naranjo ${ }^{2}$
}

Recibido: 2020-02-24 / Revisado: 2020-03-30 / Aceptado: 2020-04-05 / Publicado: 2020-07-01

Forma sugerida de citar: Ortiz-Mancero, M.F. y Núñez-Naranjo, A. F. (2021). Inteligencia Emocional: Evaluación y Estrategias en tiempos de pandemia. Retos de la Ciencia. 5(11), pp. 57-68. https://doi.org/10.53877/rc.5.11.20210701.06

\section{RESUMEN}

Este trabajo presenta un análisis de las estrategias metodológicas en función de la inteligencia emocional frente al rendimiento académico de estudiantes de educación superior durante la pandemia por covid-19. La población de estudio se conformó por 110 estudiantes. Tuvo un enfoque mixto, se realizó una investigación bibliográfica de las características y dinámica entre inteligencia emocional y rendimiento académico, se efectuó la recolección y análisis de datos mediante una batería psicológica Trait Meta-Mood Scale (TMMS) y boletines de calificaciones que permitieron describir, comprender e interpretar los fenómenos a través de las percepciones y significados producidos por las experiencias de los participantes, se aplicó la lógica deductiva de lo general a lo particular. Con los resultados obtenidos en el TMMS, se determinó que las estrategias metodológicas se centran en el desarrollo de habilidades emocionales, como un factor incidente el éxito y estabilidad social a través de la percepción, comprensión y manejo de emociones mismo que dan como resultado una apropiada relación entre el medio social y educativo. Se concluye que el contexto emocional del estudiante requiere de encontrar estabilidad emocional lo que conlleva a una mejora de su rendimiento académico.

Palabras clave: inteligencia emocional, conducta emocional, rendimiento académico.

\footnotetext{
${ }^{1}$ Magister en Educación, con mención en innovación y liderazgo educativo. Docente de nivelación y admisión Universidad Nacional de Chimborazo. Ecuador. Email: mateo.ortiz@unach.edu.ec / ORCID: https://orcid.org/0000-0001-7124-1627

2 Magister en Gestión de Proyectos Socio Productivos. Docente en la Facultad de Ciencias Humanas de la Educación y Desarrollo Social, Carrera de Educación Básica; Docente en la Maestría en Educación Mención Innovación y Liderazgo Educativo, Especialista en Pedagogía Universidad Tecnológica Indoamérica. Ecuador. Email: fernandanunez@indoamerica.edu.ec / ORCID: https://orcid.org/0000-0001-7431-2339
} 


\section{ABSTRACT}

This paper presents an analysis of methodological strategies in terms of emotional intelligence versus academic performance of higher education students during the COVID-19 pandemic. The study population consisted of 110 students. It had a mixed approach, a bibliographic research of the characteristics and dynamics between emotional intelligence and academic performance was carried out, data collection and analysis were performed by means of a psychological material Trait Meta-Mood Scale (TMMS) and report cards that allowed describing, understanding and interpreting the phenomena through the perceptions and meanings produced by the experiences of the participants, the deductive logic was applied from the general to the particular. With the results obtained in the TMMS, it was determined that the methodological strategies are focused on the development of emotional skills, as an incident factor for success and social stability through the perception, understanding and management of emotions, resulting in an appropriate relationship between the social and educational environment. It is concluded that the student's emotional context requires finding emotional stability which leads to an improvement in their academic performance.

Keywords: emotional intelligence, emotional conduct, academic performance.

\section{INTRODUCCIÓN}

La inteligencia emocional (IE) se origina de la necesidad de responder a la interrogante "¿por qué hay personas que se adaptan mejor que otras a diferentes situaciones de la vida diaria?" (Trujillo y Rivas, 2015). El identificar que los seres humanos tienen la necesidad de comunicarse y tratar de lograr los objetivos planteados a corto o largo plazo, da paso a que estas necesidades estén sujetas a emociones y sentimientos de satisfacción, esto, quiere decir que las emociones y sentimientos se relacionan entre sí, a la hora de conseguir todo lo que el cerebro y el entorno se ha propuesto (Cano Murcia y Zea Jiménez, 2012), por lo que los educadores deben contar con un amplio vocabulario emocional que procure ayudar a los alumnos a mirar en su interior para descubrir cuáles son sus estados emocionales y por qué están provocados (Bisquerra, 2005).

Fragoso-Luzuriaga (2015) menciona que es necesario diferenciar las competencias emocionales de los estudiantes que ingresan al sistema educativo superior, comprenderlas y diferenciar las emocionales para fortalecer procesos de permanencia y titulación. Alzina y Escoda (2007) decriben a la inteligencia emocional, como el conjunto de conocimientos, capacidades, habilidades y actitudes necesarios para tomar conciencia, comprender, expresar y regular de forma apropiada los fenómenos emocionales, con la finalidad de aportar un valor añadido a las funciones profesionales y promover el bienestar personal y social.

Alvarez, Pena y Losada (2017) relacionan que mejorar el bienestar de los orientadores a través de su inteligencia emocional se requiere describir el grado de relación de la inteligencia emocional con el nivel de bienestar laboral y vital, así Ramírez (2014) indica que los orientadores con altos niveles de inteligencia emocional poseen un recurso personal que les ayuda a sentirse ilusionados, motivados e implicados con su labor, de esta menara, la clave para el éxito en liderazgo es el resultado de interacciones sociales y no únicamente temas de desempeño operativo, de aquí que se supone que aquellos líderes con mayor desarrollo habilidades sociales 
y por ende mayor grado de inteligencia emocional pueden conducir de mejor manera a sus seguidores y a la organización a alcanzar sus objetivos propuestos en todo ámbito que genere una interacción social (Nájera, 2016).

Desde el 2008 en Ecuador, la normativa expresa que la educación superior tiene como finalidad la formación académica y profesional con visión científica y humanista; la investigación científica y tecnológica; la innovación, promoción, desarrollo y difusión de los saberes y las culturas; la construcción de soluciones para los problemas del país, en relación con los objetivos del régimen de desarrollo (Asamblea Nacional del Ecuador, 2008), de igual manera se establece que el nivel de formación con sujeción al Reglamento de Régimen Académico expedido por el Consejo de Educacion Superior (2014), se organiza mediante carreras, licenciaturas y afines formando profesionales capaces de analizar, planificar, gestionar y evaluar modelos y estrategias de intervención en los campos profesionales asociados a las ciencias básicas, sociales, de la educación, de la salud, humanidades y arte, así, la inteligencia emocional juega un papel central entre las interacciones de los actores y observadores de las organizaciones, puesto que en los últimos años que se ha despertado el interés en el estudio de la inteligencia emocional por parte de las empresas (Brito, 2016).

La inteligencia emocional se percibe como factor protector de conductas problémicas como la violencia, impulsividad y el desajuste emocional (FernándezBerrocal y Extremera, 2003), que se asocia con la disminución de conductas de riesgo para la salud entre las que se incluyen el consumo de tabaco y alcohol (Jimenéz, 2018). López, Megías, Leal, Gutiérrez y Fernández (2018) resaltan que las personas con una falta de habilidades emocionales recurren al consumo de drogas como una forma externa de autorregulación para disminuir, ocultar o ignorar sus estados emocionales negativos o para generar estados emocionales más agradables.

Según Bosada (2020) toda crisis, desde guerras hasta pandemias como la que se vive actualmente por el COVID-19, conlleva fuertes respuestas emocionales negativas, como pánico, estrés ansiedad, rabia y miedo. Desarrollar en las personas habilidades de aprendizaje socioemocional ayuda a que las situaciones estresantes se aborden con calma y con respuestas emocionales equilibradas. Además, estas competencias permiten fortalecer el pensamiento crítico para tomar decisiones mejor informadas en la vida, si no se atiende al desarrollo emocional de forma apropiada lo más probable es que haya un incremento de ansiedad, estrés, depresión, consumo de sustancias, comportamiento sexual de riesgo, impulsividad descontrolada, violencia.

La pandemia que se está atravesando por COVID-19 ha hecho que los estudiantes tengan que lidiar con sentimientos de aislamiento, frustración, aburrimiento, ansiedad y estrés; esto trae consigo una respuesta de desesperanza, depresión y enojo. Según Duan y Zhu (2020) Los jóvenes-adultos de 20 a 30 años no saben identificar sus emociones y por ende, han sido más susceptibles a los cambios que la pandemia por COVID-19 ha provocado; no hay un entendimiento acerca de la función real de la inteligencia emocional. Desde algunos estudios psicológicos, se ha visto que el estado de alarma nacional derivado de la epidemia del COVID - 19, genera en muchos, emociones como el miedo, la ansiedad, el estrés y el malestar emocional en un contexto de incertidumbre y de sobreinformación.

Según Dussel y Pulfer (2020) la pandemia de la COVID ha sido difícil, no solo por el riesgo del contagio, sino por la incertidumbre de no saber cuándo terminará y si se podrá vivir de nuevo sin miedo. En un libro reciente sobre la situación de la educación 
en tiempos de la pandemia, hecho a gran velocidad por docentes iberoamericanos, se resalta que el cierre de las escuelas es un acontecimiento traumático para los actores educativos; no se sabe a ciencia cierta cuáles serán los efectos, si al regresar se reinstalarán las viejas prácticas y se volverá a la "vieja normalidad" o la "nueva normalidad" será beneficiosa para innovar y romper inercias, y si el sistema se reinventará o, como lo que mencionan, permanecerá la "escuela profunda" caracterizada por la desigualdad.

Según Boyantzi, Goleman, y Rhee (2000), el rendimiento académico de los escolares tradicionalmente se ha asociado a la capacidad intelectual previa que permitiera la posibilidad de obtener buenas calificaciones. Actualmente se considera que el equilibrio personal es un factor clave para lograr un mejor rendimiento. En este sentido, conocer las propias emociones y saber restaurarlas ante las dificultades serán aspectos de importancia, así, la inteligencia emocional es uno de los aspectos relevantes a tener considerar en las habilidades y capacidades de las personas, que favorece y facilita la consecución de metas en el individuo en su tarea vital, y actúa como buena pre-dictora de la adaptación de una persona al medio.

Según Gonzalez (2020), el COVID-19 muestran una disminución de la motivación y del rendimiento académico con un aumento de la ansiedad y dificultades familiares, que genera reacciones diversas: iniciando con el miedo, enojo por el confinamiento, desconfianza, angustia, duelo por los seres queridos enfermos o que fallecieron por el contagio, lo que es un desencadenante de bajos niveles de autoestima, esfuerzo académico, laboral e interpersonal.

\section{DESARROLLO}

La inteligencia emocional para Goleam (1995) y Goleam (1998) es la capacidad para escoger las mejores opciones en la búsqueda de una solución, en cualquier caso, la inteligencia aparece relacionada con la capacidad de entender y elaborar información para usarla de manera adecuada. La inteligencia emocional es la que guía a diario para enfrentar las situaciones del contexto, mientras que en el ámbito educativo es la base esencial para el desarrollo de las capacidades en la vida diaria (Velasco Bernal, 2014).

Los inicios de la inteligencia emocional son postulados por parte de Alfred Binet en 1905 con la creación del primer test de inteligencia psicométrica con el cual se identifica que alumnos tienen éxito en sus vidas, a pesar que este modelos ha sido criticado por Sternberg y Gardner con nuevas concepciones de inteligencia es así que Sternberg en 1997 considera que hay que ir más allá del cociente intelectual y más allá de la inteligencia analítica, ya que ésta no es suficiente para tener un futuro de éxito (Jimenéz, 2018).

Barbosa y García (2018) plantean la hipótesis de que los alumnos con mayor nivel de inteligencia emocional muestran a lo largo del curso menores sentimientos de agotamiento, cinismo, estrés y mayores actitudes positivas hacia sus tareas mayor nivel de vigor dedicación y absorción, efectos positivos que explica la relación positiva y significativa en los estudios mismos que vinculan la inteligencia emocional con el rendimiento académico, mientras que Sigcha (2019) determina que existe una correlación directamente proporcional entre la inteligencia emocional y la conducta prosocial.

Educar con inteligencia emocional en los centros educativos implica que los docentes identifiquen los sentimientos y emociones, sepa controlar la expresión, no reprimir sino ofrecer modelos adecuados de expresión cuando se trata de emociones 
negativas que suelen ser más difíciles de comunicar de una forma respetuosa (Ibarrola L. De Davalillo, 2013). Evidentemente la educación de las emociones requiere una formación inicial pero también una formación permanente (Velasco Bernal, 2014). Este tipo de educación es importante, ya que se convierte en una prevención de estrés, depresión, conflictos interpersonales y a la vez potencia su desarrollo como persona (Alzina, Esnaola y Sarasa, 2015).

Importancia de la inteligencia.- Una de las posturas más conocidas de inteligencia emocional es la de Bisquerra (2005) pues indica que todo educador debe enseñar un amplio vocabulario emocional, procura ayudar a sus alumnos a mirar en su interior para descubrir cuáles son sus estados emocionales y por qué están provocados, de esta manera el alumnado comprende que las emociones son una parte fundamental del ser humano determinando el comportamiento, manifestaciones a través del ajuste social, el bienestar y la salud del individuo (Alzina, Esnaola y Sarasa, 2015).

Cada vez más niños no reciben en la vida familiar un apoyo seguro para transitar por la vida y que muchos padres no pueden ser modelos de inteligencia emocional para sus hijos, por lo que las escuelas pasan a ser el único lugar hacia donde pueden volverse las comunidades en busca de pautas para superar las deficiencias de los niños en la aptitud social y emocional (López y González, 2016), por lo que se requiren dos cambios importantes: que el profesorado comprenda que educar es mucho más que transmitir conocimientos y que la familia y los miembros de la comunidad se involucren más profundamente con la actividad escolar (Alzina, Esnaola y Sarasa, 2015).

Para el psicólogo estadounidense Howard y Gardner la inteligencia le da un sentido más amplio tomando en consideración diferentes tipos de inteligencia siendo, estas: inteligencia musical, inteligencia cenestésica corporal, inteligencia lógicomatemática, inteligencia lingüística, inteligencia espacial, inteligencia interpersonal, inteligencia intrapersonal (Herrera, 2018).

Aportes teóricos inteligencia emocional. - Para Salovey, Woolery y Mayerm la inteligencia emocional tiene tres ámbitos de expresión para las emociones, regulación de las emociones y utilización de la información de carga emocional en el pensamiento y la actuación (López y González, 2016). Por otra parte, Dulewicz y Higgs 1998, encuentran seis elementos en los cuales se relata la autoconciencia, control emocional, automotivación, empatía, manejo de relaciones, comunicación interpersonal y estilo personal (López y González, 2016).

Aspectos importantes como la empatía y la percepción interpersonal sumada a la autoestima se han identificado como variables de alto significado dentro de las relaciones sociales, éxito en actividades laborales como académicas (López y González, 2016).

A lo largo de los primeros años de vida, se adquiere emociones tales como: alegría, miedo y enfado mismos que se demuestran por medio de símbolos, signos, significados que los seres humanos sienten, siempre basándose en las experiencias pasadas, en el aprendizaje, el carácter y el estado de ánimo (Cruz, Mesa y Díaz, 2019). Ciertas reacciones de comportamiento y fisiológicas que se despliegan de las emociones son innatas, mientras que otras se pueden adquirir. Finalmente, la expresión de las emociones, se enfocan en la comunicación verbal que el individuo envía sobre el sentir y pensar y la no verbal que se enfatiza con las expresiones faciales y movimientos corporales, mediante las cuales el sujeto expresa agrado o desagrado ante la emoción. La respuesta ante una emoción difiere de una persona a otra, pues cuando la persona ya ha experimentado con anterioridad ciertas emociones 
esta preparado para reconocer, interpretar y actuar de una forma acertada ante la situación que se le presente (Rodríguez Macuna, 2015).

Es importante correlacionar la comunicación verbal y no verbal, pues es de gran ayuda para enfatizar y expresar racionalmente la emoción que en el momento se está dando gracias a aquello se logra la comprensión ante otras personas para que se interprete de manera correcta (Cruz, Mesa y Díaz, 2019).

Uno de los factores que juegan un papel fundamental en el desarrollo de la inteligencia emocional es la familia, ya que la conexión que tienen en la infancia es de suma importancia para el bienestar infantil, por lo cual los padres deben expresar de manera constante amor condicional, valor y apoyar en las actividades que desarrollan los niños (Mesa Jacobo, 2015 ). La autoestima y la inteligencia emocional fortalece de manera positiva este factor, mediante el análisis e identificación de emociones y conductas que dan un valor adecuado a las fortalezas y así se mejoraran las debilidades (Trinidad Nazario, 2018).

Otro factor corresponde a la educación, el proceso educativo se enfoca más allá del desarrollo cognitivo, ya que es de suma importancia promover el desarrollo emocional, el docente es la guía fundamental en el desarrollo emocional del ser, con respecto a fortalecer las habilidades que permiten enlazar la motivación permanente de esta manera se relaciona con el entorno y en especial poseer seguridad (Mesa Jacobo, 2015 ).

Entre las propuestas más validadas que existe acerca de la inteligencia es la de Extremera, Duran y Rey (2007) quienes aportan un marco conceptual para investigar la capacidad de adaptación social y emocional de las personas, Daniel Goleman quien escribió el primer best seller el primer libro basado en la funcionalidad contextual de las emociones y el modelo propuesto por Salovey, Woolery, y Mayerm (2001) la cual se centra en las habilidades emocionales desarrolladas por medio del aprendizaje y la experiencia cotidiana, en el cual se define qué es necesario la habilidad de las personas para percibir, usar, comprender y manejar las emociones (Mellado, Sánchez Herrera, Vicente y Bermejo, 2017).

Desarrollo de habilidades en tiempos de pandemia. - Es necesario revisar el desarrollo de habilidades que en tiempos de pandemia pueden ser fortalecidos 0 debilitados.

La facilitación emocional. - es aquella en la que el individuo tiene la capacidad para generar sentimientos que facilitan el pensamiento o los procesos cognitivos frente a una circunstancia (Barbosa y García, 2018).

Comprensión emocional. - es la que integra lo que se siente dentro del pensamiento y considera lo necesario para el cambio en las emociones tanto positivas como negativas. Por lo que para los autores enlazando de forma adecuada estas habilidades se puede facilitar el crecimiento personal y el ajuste emocional dentro de los diferentes contextos (Barbosa y García, 2018).

Estrategias para el desarrollo de la habilidad emocional en pandemia. - En el uso de estrategias participativas en el aula y específicamente para el desarrollo de la inteligencia emocional, se basa en encaminar a incentivar el interés del estudiante integrando habilidades, valores, actitudes y normas, ya que esto incrementa el aprendizaje y posibilita un ambiente armónico en el aula (Tapias, 2018).

Aquel docente que solo conoce de la materia, aunque la domine a la perfección, si no aplica estrategias metodológicas no logra desarrollar la I.E. de sus estudiantes (Tapias, 2018). Para Palacios Valderrama, Alvarez Aviles, Moreira Bolaños, y Moran Flores (2017) "el desarrollo de la I.E implica necesariamente un aprendizaje activo y 
significativo a través del cual se construye un significado mediante la interacción y el diálogo para desarrollar habilidades sociales, curiosidad, reflexión y el aprovechamiento de conocimientos con el fin de tomar determinaciones y ofrecer soluciones".

Alvarez, Pena, y Losada (2017) manifiesta que el alumno adquiere habilidades no solo en las que se apropia de los conocimientos que se le imparten, sino que adquiere la habilidad de resolver problemas, con rigor científico-afectivo, aprender a llevar responsablemente y colaborar siempre con quienes interactúan en su contexto y se adapte a estar informado a partir de la lectura constante, para así llevar esa información a la expresión escrita.

El docente debe crear un ambiente acogedor en el contexto de aula, para que el estudiante se sienta cómodo y pueda cumplir con la actividad indicada, donde su papel de facilitador corresponde en incitar a la reflexión, aprendizaje autónomo y colectivo, desarrollando su independencia cognoscitiva (Tapias, 2018).

Relación entre inteligencia emocional y rendimiento académico. - García Jiménez, Alvarado y Amelia (2014), define que el rendimiento escolar es el nivel de conocimiento demostrado en un área o materia comparado con la norma de edad y nivel académico. Cavero (2019), considera que el rendimientoacdemico predice el equilibrio emocional y examina la viabilidad del constructo como factor explicativo del rendimiento académico, no como una relación directa entre I.E y logro académico, sino analiza el efecto mediador que una buena salud mental ejerce sobre el rendimiento escolar de los estudiantes (Trinidad Nazario, 2018).

Las limitaciones evidentes de tiempo y espacio obligan a los educadores a decidir qué habilidades básicas se debe enseñar en el aula y cuáles se desarrollan por los alumnos en su vida cotidiana (Alva, 2017). De allí la interrogante ¿qué es lo que necesitan aprender los estudiantes para evitar futuros problemas?, el modelo de Salovey y Mayer proporcionan un medio eficaz para determinar las habilidades emocionales básicas y crear una base emocional que sirve para la adquisición y promoción de otras competencias sociales, emocionales y afectivas más complejas (Cavero, 2019). Los programas de aprendizaje socioemocional se centran en aspectos cognitivos como es la creatividad, razonamiento, solución de problemas, mientras que otros se centran solamente en habilidades sociales como es la asertividad, presión de grupo, manejo de conflictos (Alva, 2017).

La inteligencia emocional se enseña en los sistemas básicos de educación siendo adecuado cualquier contexto y su aprendizaje es necesario a lo largo del ciclo de vida, no obstante las habilidades emocionales básicas son introducidas desde la escuela a partir de los tres años de edad e influye de manera decisiva en la adaptación social y psicológica de los estudiantes, en su bienestar emocional, e incluso en sus logros académicos y en su futuro laboral (Cavero, 2019).

Estrategias para fortalecer la IE en pandemia. -Comprender el estilo de aprendizaje y recompensa que este genera al momento de evocar lo aprendido, las bases primordiales de esto son cómo, el estudiante ha captado el mensaje, como siente la actividad, es decir si la percibe como aburrida, cansada, tediosa, o si la observa como un reto, algo diferente a lo que mientras mejor responde, mejor recompensa tiene académicamente.

El docente debe conocer y manejar un vocabulario emocional amplio, en pro de generar en el estudiante confianza, con la tendencia de reconocer todo lo que este brinda, como positivo, olvidarse por un momento de la clásica evaluación cualitativa, 
sumativa, que se enfoca en el valor que un estudiante obtiene al repetir información redundante de la web.

Las circunstancias de aprendizaje son difíciles por la condición de conectividad en todo el mundo, lo que genera emociones de frustración y cuando esto sucede el estudiante, frena su motivación por entender, aprender, asimilar los contenidos expuestos, mira su posición y cree no poder competir en el mundo académico por la falta de recursos, aquí es donde entra el aspecto de innovar y manejar la tendencia del que no importan los medios, sino la producción de la creatividad a través de diferentes caminos, se refiere a que las tareas están encaminadas al uso exclusivo de herramientas tecnológicas, desde niños se tiene contacto con el método pictográfico, donde se puede representar la percepción del mundo, se debe olvidar la limitación que las tics dan y se debe empezar por desarrollar la capacidad creativa del estudiante, reconociendo esto como la cumbre de la educación, así denota el maestro actual, constructivista, que parte de un currículo flexible (Bosada, 2020).

No obstante, hay que reconocer la valides de los recursos tecnológicos, más aun, algunas aplicaciones que sirven para mejorar el reaprender que tiene un estudiante, desde el método lúdico, a través de juegos donde la actividad se vuelva atractiva y a su vez significativa, es así que las herramientas como Kahoot, Genially, ponen de manifiesto la estrecha relación que existe entre la estructura mental y la actividad lúdica, confirmada en la evolución del juego que se va dando en el individuo desde los más elementales juegos sensorio motrices a complejos juegos de reglas que contienen muchas de las normas sociales y morales propias de la sociedad adulta (Tovar, 2017).

Estrategia de repetición. - consiste en pronunciar, nombrar o decir de forma repetida los estímulos presentados dentro de una tarea de aprendizaje. Se trataría, por tanto, de un mecanismo de la memoria que activa los materiales de información para mantenerlos en la memoria a corto plazo y, a la vez, transferirlos a la memoria a largo plazo. Por otro lado, mientras que la estrategia de elaboración trata de integrar los materiales informativos relacionando la nueva información con la información ya almacenada en la memoria, la estrategia de organización intenta combinar los elementos informativos seleccionados en un todo coherente y significativo. Además, dentro de esta categoría de estrategias cognitivas también estarían las estrategias de selección o esencialización, cuya función principal es la de seleccionar aquella información más relevante con la finalidad de facilitar su procesamiento (Tapias, 2018).

Estrategias metacognitivas. - son un conjunto de estrategias que permiten el conocimiento de los procesos mentales, así como el control y regulación de estos con el objetivo de lograr determinadas metas de aprendizaje, el conocimiento metacognitivo requiere consciencia y conocimiento de variables de la persona, de la tarea y de la estrategia (Caramelo, 2017). Se debe desarrollar en forma consciente y sistemática las habilidades socioemocionales que necesitan los estudiantes para afrontar situaciones inciertas y desconcertantes que actualmente enfrentan ante la pandemia por COVID-19.

La importancia de los componentes afectivo-motivacionales en la conducta estratégica es puesta de manifiesta por la mayor parte de los autores que trabajan en este campo. Todos coinciden en manifestar que los motivos, intenciones y metas de los estudiantes determinan en gran medida las estrategias específicas que utilizan en tareas de aprendizaje particulares. Por eso, entienden que la motivación es un 
componente necesario de la conducta estratégica y un requisito previo para utilizar estrategias (Caramelo, 2017).

Según Bosada, (2020), para mejorar la formación de los docentes en el ámbito socioemocional se deben incluir las habilidades socioemocionales en los programas de formación docente, tanto en la formación inicial como en la práctica de la docencia, y en programas de desarrollo profesional, a fin de abordar el estrés e impulsar las competencias emocionales y sociales en el aula, garantizar que los directores de los centros educativos asignen tiempo a los docentes para su formación socioemocional y les den la oportunidad de adquirir estas habilidades y promover la comunicación y la creación de redes entre docentes. Es decir, crear comunidades de prácticas entre el profesorado dentro de los centros educativos y entre escuelas distintas para fomentar el aprendizaje de las habilidades socioemocionales, el apoyo mutuo y el bienestar continuos. Hay que tener en cuenta que, "el docente posibilita la comunicación multidireccional en el intercambio de ideas, experiencias, conocimientos, emociones, sentimientos y pasiones en función de la reciprocidad y capacidad de impacto social mutuo entre docentes y educandos, a partir de la cultura, como vínculo estético primordial." (Castillo, 2015, p. 199)

\section{MÉTODO}

Este trabajo se basó en un enfoque mixto, se realizó una investigación bibliográfica de las características y la dinámica entre I.E y rendimiento académico, se efectuó la recolección y análisis de datos mediante una batería psicológica Trait Meta-Mood Scale (TMMS IV) y boletines de calificaciones que permitieron describir, comprender e interpretar los fenómenos a través de las percepciones y significados producidos por las experiencias de los participantes, se aplicó la lógica deductiva de lo general a lo particular. La población considerada estuvo constituida por 156 estudiantes de los primeros semestres de la carrera Pedagogía de las Artes de la UNACH, acorde a los listados obtenidos por parte de la secretaria de carrera perteneciente al periodo académico 2020.

\section{RESULTADOS}

Los resultados obtenidos mediante la aplicación del test TMMS-24 se basó en el Trait Meta- Mood Scale del grupo de investigación de Salovey y Mayer que está conformada por 24 ítems que valora tres factores: percepción, comprensión y regulación de la emoción (Villacreces \& Achi, 2017). En el cual de 50 estudiantes hombres que representan el 100\%; el 59\% posee un nivel adecuado de percepción emocional; el $21 \%$ se ubica en un nivel bajo de percepción emocional; y el $20 \%$ se sitúa en un nivel alto de percepción emocional y de 60 estudiantes mujeres que representan el 100\%; el $62 \%$ posee un nivel adecuado de percepción emocional; el $26 \%$ se ubica en un nivel bajo de percepción emocional; y el $12 \%$ se sitúa en un nivel alto de percepción emocional.

En cuanto a la comprensión emocional de 50 estudiantes varones que representan el 100\%; el $41 \%$ se sitúa en un nivel alto de comprensión emocional.; el $53 \%$ posee un nivel adecuado de comprensión emocional; y el $6 \%$ se ubica en un nivel bajo de comprensión emocional, en cuanto a las mujeres de 60 estudiantes mujeres que representan el 100\%; el $49 \%$ posee un nivel adecuado de comprensión emocional; el 31 \% se ubica en un nivel bajo de comprensión emocional; y el 20 \% se sitúa en un nivel alto de comprensión emocional. 


\section{DISCUSIÓN}

Educar con inteligencia emocional implica que el profesorado identifique los sentimientos, emociones, control de la expresión, ofreciendo modelos adecuados para la expresión sobre todo de emociones negativas que suelen ser las más difíciles de comunicar (Rodríguez Macuna, 2015). Siendo así que se exige dos cambios importantes que el profesorado comprenda que educar es mucho más que transmitir conocimientos y que la familia y los miembros de la comunidad se involucren profundamente con la actividad escolar mejorando de esta manera la asimilación de los conocimientos y mejorando el rendimiento académico (Alzina, Esnaola y Sarasa, 2015.

Las personas que poseen habilidades emocionales tienden a ser más productivas y efectivas en las actividades que emprenden, motivarse a sí mismo una emoción tiende a impulsar hacia una acción, por eso la emoción y motivación están íntimamente interrelacionados (García-Fernández y Jiménez-Mas, 2010).

Finalmente, en conclusión, cabe señalar que la investigación determinó que existe una correlación ente la inteligencia emocional y el rendimiento académico de los estudiantes de la carrera de de pedagogía de las artes en la Universidad Nacional de Chimborazo, lo que indicaría que la inteligencia emocional y el rendimiento académico guardan una relación recíproca y directa, así que aquel que tiene una alta inteligencia emocional tiende a tener un buen rendimiento académico y por el contrario quien presenta una inteligencia emocional baja tiende a tener un bajo rendimiento académico.

\section{REFERENCIAS BIBLIOGRÁFICAS}

Alva, M. C. (2017). Autoestima, hábitos de estudio y rendimiento académico en estudiantes universitarios. Dialnet Propósitos y Representaciones.

Alvarez, M., Pena, M., \& Losada, L. (2017). "Mission Possible: Improve the Welfare of Counselors through Their Emotional . Revista Española de Orientación y Psicopedagogía.

Alzina, L., Esnaola, I., \& Sarasa, M. (2015). European Journald of Education and Psychology Predictive Capacity of Social Support on Emotional Intelligence in Adolescence. European Journal of Education and Psychology, 23-29.

Alzina, R. B., \& Escoda, N. P. (2007). Las Competencias Emocionales. Facultad de Educación. UNED , 61-82.

Asamblea Nacional del Ecuador. (2008). Ley Organiza de Educacion Superior(LOES). Quito: SITEAL.

Barbosa, S. H., \& García, M. A. (2018). Inteligencia emocional y control de estrés. RepositorioUniversidad Cooperativa de Colombia.

Bisquerra, A. R. (2005). La Educación Emocional En La Formación Del Profesorado. Revista Interuniversitaria de Formación Del Profesorado, 95.

Bosada, M. (27 de Mayo de 2020). Educaweb. Obtenido de Educaweb: https://www.educaweb.com/noticia/2020/05/27/educacion-emocional-claveensenanza-aprendizaje-tiempos-coronavirus-19205/

Boyantzi, Goleman, \& Rhee. (2000). Custering competence in emotional intelligence.Insights from the emoyional competence inventory. Jossey-Bass. 
Brito, J. G. (2016). Inteligencia Emocional y Liderazgo. INNOVA Research Journal, 16-21.

Castillo-Bustos, M. R. y Montoya-Rivera, J. (2015). Dinámica ideo-espiritual de la formación estético-pedagógica del docente. Alteridad, 10(2), pp. 190-204.

Cano Murcia, S. R., \& Zea Jiménez, M. (2012). Manejar las emociones, factor importante en el mejoramiento de la calidad de vida. Revista Logos, Ciencia \& Tecnología, 58-67.

Caramelo, Á. M. (2017). Estrategias de aprendizaje e inteligencia emocional y su relacion con el rendimiento académico . Repositorio Universidad Internacional de la Rioja UNIR.

Cavero, M. Á. (2019). Inteligencia emocional y rendimiento académico en alumnos de educación secundaria obligatoria. Facultad de Educación Universidad de Zaragoza, España.

Consejo de Educacion Superior (CES). (2014). Reglamento de Regimen Academico. Quito.

Cruz, R. G., Mesa, M. C., \& Díaz, M. B. (2019). Convivencia y bienestar: categorías necesarias para la educación positiva. Revista Científica de la Universidad de Cienfuegos.

Duan, \& Zhu. (2020). Psychological interventions for people affected by the COVID-19 epidemic. The Lancet Psychiatry, .

Dussel, Ferrante, \& Pulfer. (2020). Revista digital de la Universidad autonoma de Chiapas. Obtenido de Revista digital de la Universidad autonoma de Chiapas: http://redesib.formacionib.org/grupos/docentes-frente-a-la-pandemia/blog/pensa

Extremera, N., Duran, A., \& Rey, L. (2007). Inteliegencia emocional y su relacion con los niveles de Burnout, engagement y estres en estudiantes universitarios. España: Revista de Educacion.

Fernández-Berrocal, P., \& Extremera, N. (2003). Relation of perceived emotional intelligencegence and health-related quality of life in middle-aged women. Psychological Reports, 47-59.

Fragoso-Luzuriaga, R. (2015). Inteligencia emocional y competencias emocionales en educación superior, ¿un mismo concepto? Revista iberoamericana de educación superior, 10-25.

García Jiménez, M. V., Alvarado, I., \& Amelia, J. B. (2014). La predicción del rendimiento académico: regresión lineal versus regresión logística . Journal Scholar Metrics.

García-Fernández, M., \& Jiménez-Mas, S. I. (2010). La inteligencia emocional y sus principales modelos: propuesta de un modelo integrador. Revista Digital del Centro del Profesorado Cuevas-Olula(Alemania).

Goleam, D. (1995). Emotional Intelligence. New York: Bantam: .

Goleam, D. (1998). Emotional Intelligence. New York: Bantam: .

Gonzalez, L. (15 de Octubre de 2020). Revista digital de la universidad autonoma de Chiapas. Obtenido de Revista digital de la universidad autonoma de Chiapas: https://espacioimasd.unach.mx/index.php/Inicio/article/view/249/782

Herrera, M. C. (2018). El refuerzo pedagógico como herramienta para el mejoramiento de los aprendizajes. Repositorio Universidad Andina Simón Bolívar .

Ibarrola L. De Davalillo, B. (2013). Dirigir y educar con Inteligencia Emocional. Ponencia del VII Congreso de Educación y Gestión .

Jimenéz, A. J. (2018). Inteligencia emocional. AEPap. Curso de Actualiazion Pediatria.

López, M. S., Megías, A., Leal, R. G., Gutiérrez, M., \& Fernández, P. (2018). Relación entre la inteligencia emocional percibida y el comportamiento de riesgo en el ámbito de la salud. Escritos de Psicología .

López, R. B., \& González, M. (2016). Rendimiento Académico y Autopercepcion de Inteligencias Multiples e Inteligencia Emocional. Revista Electrónica "Actualidades Investigativas en Educación".

Mesa Jacobo, J. R. (2015 ). Inteligencia Emocional, Rasgos de Personalidad e Inteligencia Psicométrica en Adolescentes . Universidad de Murcia - Facultad de Psicología - tesis 
Nájera, S. (2016). Liderazgo e Inteligencia Emocional. INNOVA Research Journal.

Palacios Valderrama, W. N., Alvarez Aviles, M. E., Moreira Bolaños, J. S., \& Moran Flores, C. (2017). Una mirada al pensamiento crítico en el proceso docente educativo de la educación superior. EDUMECENTRO.

Ramírez, M. d. (2014). La influencia de la inteligencia emocional en el trabajo del orientador educativo y laboral. Dialnet, Avances en Ciencias de la Educación y del Desarrollo.

Rodríguez Macuna, L. M. (2015). Inteligencia Emocional y Aprendizaje en la Infancia. Universidad Pedagógica y Tecnológica De Colombia - Repositorio.

Salovey, P., Woolery, A., \& Mayerm, J. B. (2001). Emotional Intelligence: Conceptualization and Measurement - Chapter Eleven. University of New Hampshire- Scholars Repository.

Sigcha Paredes, A. P. (2019). DSpace Universidad Indoamerica. Obtenido de http://repositorio.uti.edu.ec/bitstream/123456789/1259/1/PROYECTO\%20DE\%20INV ESTIGACI\%c3\%93N\%20SIGCHA\%20ALISSON.pdf

Supervía, P. U., \& Bordás, C. S. (2018). Motivación escolar, inteligencia emocional y rendimiento académico en estudiantes de educación secundaria obligatoria. Revista UCR.

Tapias, M. G. (2018). Estilos de aprendizaje, estrategias para enseñar. su relación con el desarrollo emocional y "aprender a aprender". Repositorio Universidad de Valladolid, España. .

Tovar, M. (2017). El método lúdico y su influencia en el desarrollo de habilidades cognitivas . En M. Tovar, El método lúdico y su influencia en el desarrollo de habilidades cognitivas (pág. 40). Lima .

Trinidad Nazario, N. Y. (2018). La inteligencia emocional y el rendimiento académico en alumnos del tercer ciclo del programa academico de Psicología de la Universidad de Huánuco 2017- II. Repositorio Universidad de Huánuco.

Trujillo Flores, M. M., \& Rivas Tovar, L. A. (2015). Orígenes, evolución y modelos de inteligencia emocional. INNOVAR. Revista de Ciencias Administrativas y Sociales, 924.

Velasco Bernal, J. J. (2014). La Inteligencia Emociona. Industrial Data , 80.

Vera, R. (2014). Universidad Tecnica de Ambato Facultad de Ciencias de la Salud Carrera de Terapia Física.

Villacreces, J. A., \& Achi, V. H. (2017). Aplicación del test TMMS-24 para el análisis y descripción de la Inteligencia Emocional considerando la influencia del sexo. 